\title{
Mode of action of trifluorothymidine (TFT) against DNA replication and repair enzymes
}

\author{
NORIHIKO SUZUKI ${ }^{1}$, TOMOHIRO EMURA ${ }^{2}$ and MASAKAZU FUKUSHIMA ${ }^{1}$ \\ ${ }^{1}$ Tokushima Research Center, Taiho Pharmaceutical Co., Ltd., 224-2 Hiraishi-ebisuno, Kawauchi, Tokushima 771-0194; \\ ${ }^{2}$ The Second Cancer Laboratory, Taiho Pharmaceutical Co., Ltd., 1-27 Misugidai, Hanno-city, Saitama 357-8527, Japan
}

Received December 24, 2010; Accepted January 31, 2011

DOI: $10.3892 /$ ijo. 2011.1003

\begin{abstract}
Trifluorothymidine (TFT) is well known to be converted to TFT-monophosphate by thymidine kinase and to inhibit thymidylate synthase. In addition, TFT-triphosphate (TFT-TP) is also incorporated into DNA, resulting in cytocidal effects. However, the precise mechanism of TFT-induced DNA damage is still unclear. Therefore, we investigated the modes of action of TFT against DNA replication and repair enzymes, as compared with those of 5FU and FdUrd. When HeLa cells were treated with TFT at a concentration of $1 \mu \mathrm{M}$ ( $\mathrm{IC}_{50}$ value), the concentration of TFT in the DNA was calculated as $62.2 \pm 0.9 \mathrm{pmol} / 1 \times 10^{6}$ cells for $4 \mathrm{~h}$. On the other hand, following treatment of the cells with FdUrd $(0.5 \mu \mathrm{M})$ and $5 \mathrm{FU}(10 \mu \mathrm{M})$ at their $\mathrm{IC}_{50}$ doses, the drug concentrations in the DNA were 7.53 , and $0.17 \mathrm{pmol} / 1 \times 10^{6}$ cells for $4 \mathrm{~h}$, respectively. These results show the markedly greater degree of incorporation of TFT into the DNA of the HeLa cells compared with that of 5FU (approximately more than 300-fold for $4 \mathrm{~h}$ ) or FdUrd (approximately more than 8 -fold for $4 \mathrm{~h}$ ). The primer extension assay demonstrated that TFT-TP was also incorporated into the T-sites of the growing DNA strand, however, it competed only weakly with thymidine triphosphate. The DNA glycosylase assay was performed using commercially available DNA glycosylase and fractionated HeLa cell extracts obtained by gel filtration. There was no detectable excision of the TFT pairing to adenine by uracil DNA glycosylase (UDG), thymine DNA glycosylase (TDG), methyl-CpG binding domain 4 (MBD4) or the fractionated HeLa cell extracts, however, TDG and MBD4 were able to excise the TFT pairing to guanine. Additional data indicate that small-interfering RNA-mediated knockdown of TDG or MBD4 significantly increased the resistance to the cytotoxic effects of FdUrd, but not to that of
\end{abstract}

Correspondence to: Dr Norihiko Suzuki, Tokushima Research Center, Taiho Pharmaceutical Co., Ltd., 224-2 Hiraishi-ebisuno, Kawauchi, Tokushima 771-0194, Japan

E-mail: n-suzuki@taiho.co.jp

Key words: TFT, trifluorothymidine, 5-trifluoromethyl-2'deoxyuridine, DNA replication, DNA repair, 5-fluorouracil, 5-fluoro-2'-deoxyuridine
TFT. These studies show the greater degree of incorporation of TFT into the DNA than that of 5FU or FdUrd, and that such a high degree of incorporation of TFT residues into the DNA might be related to exhibit potent cytotoxic activity to be refractory to cleavage by these DNA glycosylases; thus, the DNA-directed cytotoxic effect of the compound is quite different from that of $5 \mathrm{FU}$.

\section{Introduction}

The antimetabolite 5-fluorouracil (5FU), which is frequently used to treat gastric, colorectal, head/neck, breast and other cancers, is thought to exert its anti-tumor activity by impairing RNA function following its incorporation into the RNA as 5-fluoro-2'-uridine-5'-triphosphate (FUTP), or by inhibiting thymidylate synthase (TS). Although RNA incorporation may contribute to its cytotoxicity under certain conditions (1), damage to DNA is generally considered to be the principal mechanism of tumor cell killing by 5FU. The therapeutic importance of direct action of 5FU on the DNA is emphasized by a direct correlation of the TS activity with the response rate of tumors or cancer cell lines to treatment with 5FU (2). TS inhibition deprives the cell of the capacity to synthesize dTMP from dUMP and, thereby, elevates the cellular dUTP levels at the expense of dTTP. The resulting higher intracellular concentrations of dUTP and FdUTP overwhelm dUTPase and become available for incorporation into the genome by DNA polymerase (3).

Like 5FU and 5-fluoro-2'-deoxyuridine (FdUrd), trifluorothymidine (TFT) is also said to exert its cytocidal action by inhibiting TS (4). However, we found that when tumors were exposed to high concentrations of TFT for short periods of time, the drug was incorporated into the DNA and exerted its cytocidal activity by causing DNA fragmentation rather than by inhibiting TS (5-7). Based on these observations, TAS-102 was developed as a novel drug preparation composed of a combination of TFT and TPI (an inhibitor of thymidine phosphorylase, the enzyme involved in the biodegradation of TFT). TPI is expected to reinforce the uptake of TFT by the tumor DNA and thereby promote the drug's action against cancers that exhibit resistance or low sensitivity to 5FU due to high TS expression or induction in the tumor.

The base excision repair pathway is conserved from bacteria to humans, and is initiated by DNA glycosylases. In the first 
step of this pathway, DNA glycosylases remove the structurally altered base from the DNA backbone by the associated $\mathrm{N}$-glycosylase activity. The resulting apurinic/apyrimidinic (AP) site is then incised by the AP lyase activity of the DNA glycosylases via the $\beta$-elimination (or $\delta$-elimination) of the $3^{\prime}$ phosphodiester bond. Further processing of this repair intermediate by the sequential action of AP endonuclease, DNA polymerase and ligase restores the DNA. 5FU is a substrate for UNG, SMUG1 (8), TDG (9), and MBD4 (10) among the human DNA glycosylases, and at least some of these proteins may be expected to address the $5 \mathrm{FU} / \mathrm{G}$ and 5FU/A base pairs also in vivo.

In this study, we investigated the modes of action of TFT against DNA replication and DNA repair enzymes as compared with those of 5FU and FdUrd.

\section{Materials and methods}

Oligonucleotides. Oligonucleotide-containing TFT was synthesized enzymatically as described previously (11). Other oligonucleotides (gel-purified grade) were purchased from Sigma-Aldrich (Tokyo, Japan).

Cell culture and whole-cell extract of HeLa cells. The HeLa cell line was purchased from Dai-Nippon Pharmaceutical Co., Ltd.(Osaka, Japan). Whole-cell extracts were prepared according to the method reported by Manley et al (12).

DNA incorporation of TFT, dThd, 5FU, and FdUrd in HeLa cells. Incorporation of various deoxyribonucleosides into the DNA were measured in the acid-insoluble fraction, as described previously (5).

Primer extension assay. The DNA primer extension assay (13) was performed using the following oligomers: DNA(1), *5' [ $\left.{ }^{32} \mathrm{P}\right]-$ GACTAGTCTGCATGCC-3'; DNA(2), 5'-CATG CAACGTACGTAGGCATGCAGACTAGTC-3'. In order to assess the ability of a primer to extend past a TFT lesion in the template, the following oligomers were prepared: DNA(3), *5[ $\left.{ }^{32} \mathrm{P}\right]$ '-CATGCAACGTA-3'; DNA(4),5'-GACTAGTCTGCAT GCCXACGTACGTTGCATG-3' where $\mathrm{X}$ is either normal $\mathrm{T}$ or TFT.

DNA glycosylase assay. The DNA glycosylase assay (14) was performed using commercially available Methanobacterium thermoautotrophicum thymine DNA glycosylase and E. coli uracil-N-glycosylase (Trevigen Inc., MD, USA), and the following oligomers: DNA(4), *5'-GACTAGTCTGCATGCCXACGT ACGTTGCATG-3' where X is U, T, TFT, 5-bromodeoxyuridine (BrU)or 5FU;DNA(2), 5'-CATGCAACGTACGTAGGCATGC AGACTAGTC-3'; DNA(5), 5'-CATGCAACGTACGTGGGCA TGCAGACTAGTC-3'.

Preparation of MBD4-depleted or TDG-depleted whole-cell extract of HeLa cells by immunoprecipitation. Immunoprecipitation of MBD4 or TDG was performed using magnetic Dynabeads protein G (Dynal Biotech GmbH, Hamburg, Germany) loaded with rabbit anti-MBD4 (A301-634A, Bethyl Lab., Inc., TX, USA) or rabbit anti-TDG (BS2053, Bioworld Technology, Inc., MN, USA) antibodies, respectively.
Analysis of fractionated whole-cell extracts of HeLa cells obtained by gel filtration on Superdex 75. In order to fractionate the whole-cell extracts of HeLa cells, the HPLC system, Model LC-10 AD (Shimadzu, Kyoto, Japan), and automated fraction collector, Model FC-203 (Gilson Inc., WI, USA), were utilized. Gel filtration was carried out using the Superdex 75 column (10/300 GL, GE Healthcare, AZ, USA).

Western blot analysis. Western blot analysis was performed using anti-MBD4 or anti-TDG as described in the section of immunoprecipitation in Materials and methods.

shRNA. shRNA constructs against TDG (cat. no. TG316755) and MBD4 (cat. no. TG311558) in pGFP-V-RS vectors were obtained from Origene Technologies Inc. (MD, USA). The HeLa cells in 6-well dishes were transfected with Lipofectamine 2000 (Invitrogen, CA, USA) along with $0.5 \mu \mathrm{g}$ pGFP-V-RS vector inserted with shRNA against TDG or MBD4; cells transfected with shRNA against a target not present in mammalian cells were used as the control. Stable clones were generated in accordance with the manufacturer's recommendations.

Cell sensitivity assays. Cell viability of the HeLa cells and of the TDG- or MBD4-knockdown cells were measured using Cell Counting Kit-8 (Dojindo, Kumamoto, Japan).

\section{Results}

Incorporation of TFT, 5FU and FdUrd into the cellular DNA. Incorporation of several 5'-substituted pyrimidine nucleosides into the DNA was measured (Table I). Each drug was added at the $\mathrm{IC}_{50}$ values determined by MTT assay (data not shown). The uptake of TFT by DNA was calculated as 29.51 for $2 \mathrm{~h}$ and 62.22 for $4 \mathrm{~h}$ (pmol/1 $\times 10^{6}$ cells). Although the percent TFT incorporation into the DNA was $39-46 \%$ relative to that of thymidine (dThd), it was much more than that of 5FU [0.13 for $2 \mathrm{~h}$ and 0.17 for $4 \mathrm{~h}\left(\mathrm{pmol} / 1 \times 10^{6}\right.$ cell)] and FdUrd [5.51 for $2 \mathrm{~h}$ and 7.53 for $4 \mathrm{~h}\left(\mathrm{pmol} / 1 \times 10^{6}\right.$ cells)].

Effect of TFT on DNA polymerase $\alpha$. The ability of DNA polymerase $\alpha$ (pol $\alpha)$ to extend the primer was then evaluated in the absence and presence of TFT-TP (Fig. 1). When both dTTP and TFT-TP were omitted from the reaction mixture (Fig. 1A, lane 1), no extension of the primer was observed, since the next position was a T-site (17-mer). When various concentrations of TFT-TP were included in the T-minus reaction, pol $\alpha$ was able to use the nucleotide analogue as an alternate substrate for incorporation into the T-site. Furthermore, the enzyme stopped at the T-site (17, 21, 25, 26, 30-mer). These results suggest that TFT incorporation was associated with a pause in the progression of polymerization and that it impaired the polymerization process at the sites of its incorporation. In a complete reaction mixture that contained $30 \mu \mathrm{M}$ of each dNTP, pol $\alpha$ extended the primers to 31-mer (Fig. 1, 4N conditions-TFT-TP $0 \mu \mathrm{M}$ ).

When increasing concentrations of TFT-TP were included in the $4 \mathrm{~N}$ reactions, TFT did not obviously compete with dTTP as a substrate for pol $\alpha$ to become incorporated into the T-sites of the elongating strands, even at the concentration of $100 \mu \mathrm{M}$ (Fig. 1, 4N conditions-TFT-TP 0-100 $\mu \mathrm{M}$ ). 
Table I. Incorporation of 5'-substituted pyrimidine nucleosides into DNA.

\begin{tabular}{|c|c|c|c|}
\hline \multirow{3}{*}{ Drug } & \multirow{3}{*}{$\begin{array}{c}\text { Concentration } \\
\mu \mathrm{M}\end{array}$} & \multicolumn{2}{|c|}{ Incubation (h) } \\
\hline & & \multicolumn{2}{|c|}{$\mathrm{pmol} / 1 \times 10^{6}$ cells } \\
\hline & & 2 & 4 \\
\hline dThd & 1 & $77.48 \pm 0.97^{\mathrm{a}}$ & $134.86 \pm-7.75$ \\
\hline TFT & 1 & $29.51 \pm 0.99$ & $62.22 \pm 0.91$ \\
\hline $5 \mathrm{FU}$ & 10 & $0.13 \pm 0.01$ & $0.17 \pm 0.03$ \\
\hline FdUrd & 0.5 & $5.51 \pm 3.00$ & $7.53 \pm 0.28$ \\
\hline
\end{tabular}

The radioactivity incorporated into the acid-insoluble material was determined as described in Materials and methods. ${ }^{a}$ Mean of $n=4$ and SD.

\section{A}

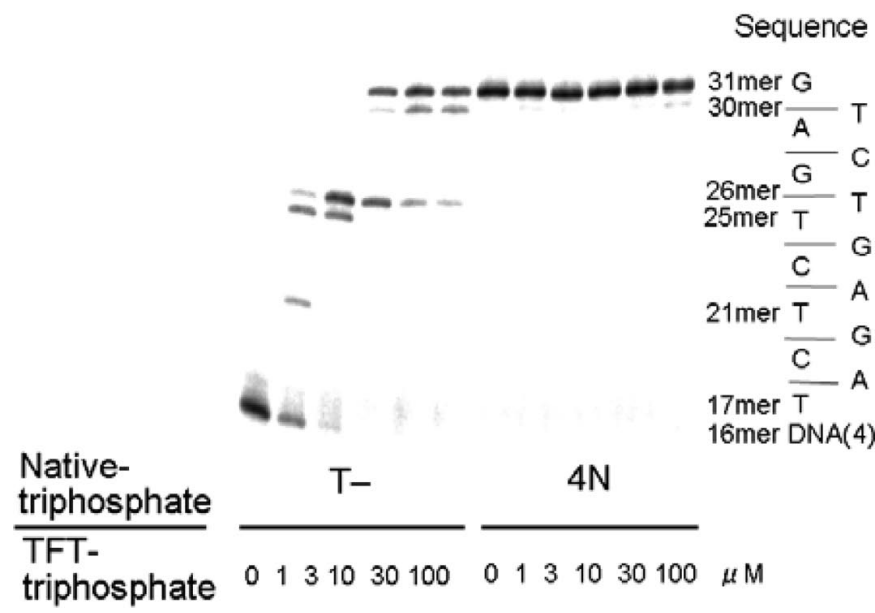

B

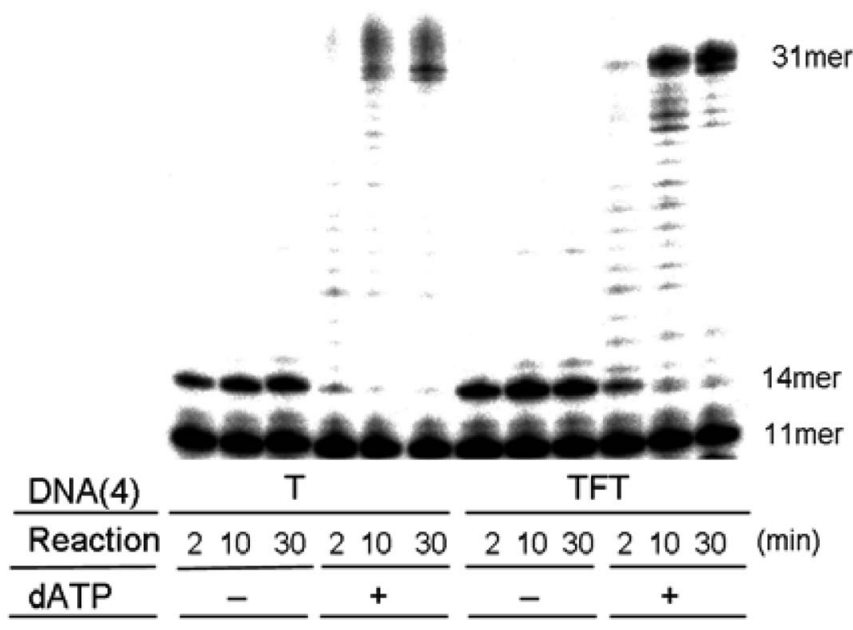

Figure 1. Action of TFT on DNA primer extension in vitro. (A) DNA elongation by DNA polymerase $\alpha$ was estimated with the $5{ }^{\prime}-{ }^{32} \mathrm{P}$-labeled primer/template [primer; DNA(1), template; DNA(2), as described in Materials and methods]. T-reactions contained $30 \mu \mathrm{M}$ each of dATP, dCTP, dGTP, without dTTP. 4N reactions contained $30 \mu \mathrm{M}$ each of dATP, dCTP, dGTP, and dTTP. (B) Activities of the primers to produce extension past a TFT lesion in the template were estimated. DNA elongation by DNA polymerase $\alpha$ was estimated with the 5'-32 P-labeled primer/template [primer; DNA(3), template; DNA(4) (X = dTTP or TFT-TP)] with or without dATP $(30 \mu \mathrm{M})$. All reactions, except for the case of dATP, contained $30 \mu \mathrm{M}$ each of dCTP, dGTP, and dTTP.
A
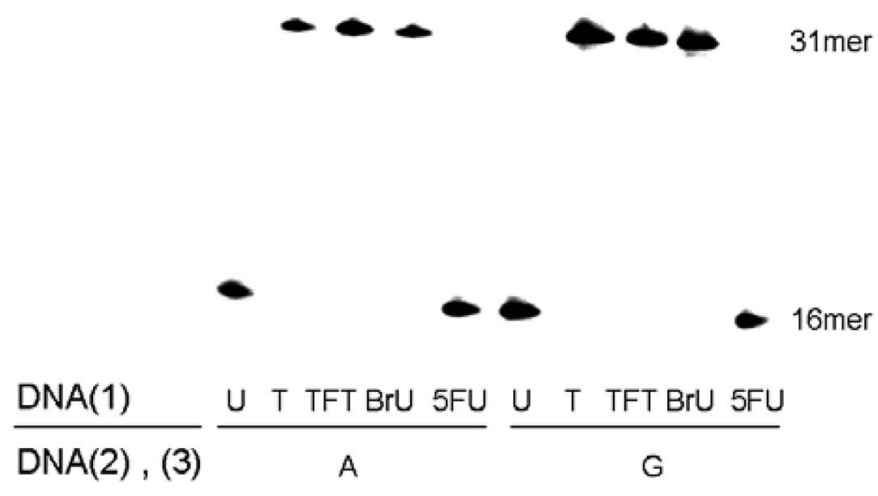

$\mathrm{B}$

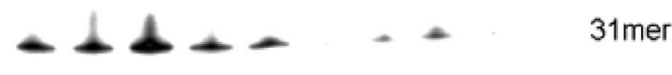

$\operatorname{DNA}(2),(3)$

U T TFT BrU 5FU

A

G

Figure 2. Cleavage of oligonucleotides containing several 5 -substituted pyrimidine nucleotide residues by uracil DNA glycosylase (A) or thymine DNA glycosylase (B). Lanes 1-5 show cleavage of a double-stranded oligonucleotide with several 5-substituted pyrimidine nucleotide residues paired to adenine, and lanes 6-10 show the same sequence with the analogues paired to guanine.

Activity of the primer to extend past a TFT lesion in the template. When dATP was deleted from the reaction mixture (Fig. 1B, lanes 1-3 and 7-9), the DNA elongation stopped at one nucleotide before the X site of the DNA(4), indicated by the arrow (13-mer). While dATP was added to the reaction mixture (Fig. 1B, lanes 4-6, 10-12), synthesis on the template containing TFT and on the control template resulted in essentially complete elongation of the primer to the full length of the template strand by the final time-point.

Excision activity by uracil DNA glycosylase and thymine DNA glycosylase. The DNA glycosylase assay was carried out using commercially available Escherichia coli uracil-DNA glycosylase (e-UNG) and Methanobacterium thermoautotrophicum mismatch thymine-DNA glycosylase (m-TDG), as shown in Fig. 2. The e-UNG excised the U/A, U/G, 5FU/A, and 5FU/G base pairs, while the TFT/A and TFT/G base pairs could not be excised by this enzyme (Fig. 2A). On the other hand, although m-TDG was not be able to excise the TFT/A base pair, just like e-UNG, the enzyme were able to excise the TFT/G base pair (Fig. 2B).

Separation and analysis by gel filtration through Superdex 75 of whole-cell extract of HeLa cells and the MBD4- and TDGdepleted extracts. In order to analyze the cleavage activity of a HeLa cell extract, analytical gel filtration was performed 
A
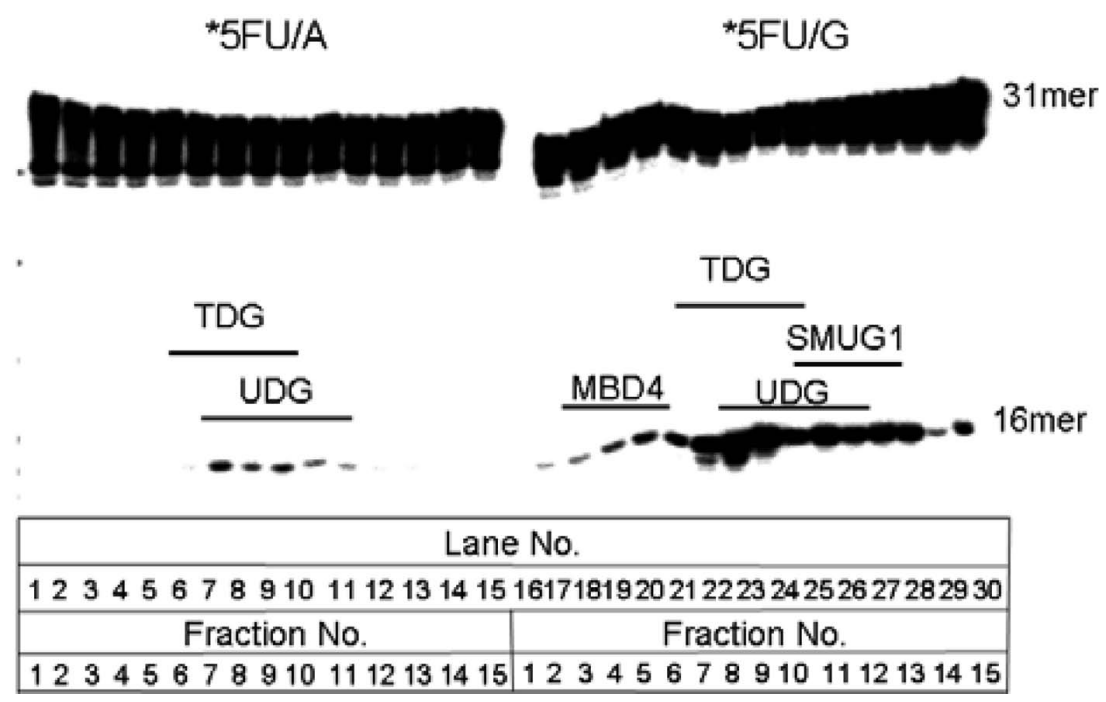

B

*TFT/A

*TFT/G

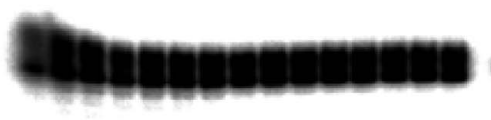

$31 \mathrm{mer}$

16 mer

Lane No.

123456789101112131415161718192021222324252627282930

Fraction No. $\quad$ Fraction No.

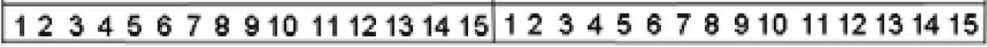

C

*TFT/A $\quad{ }^{* T F T / G}$
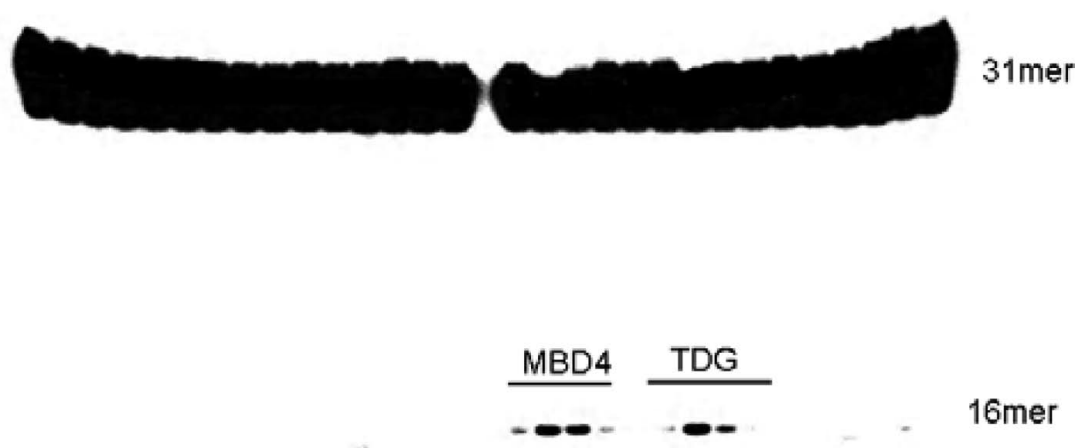

Lane No.

123456789101112131415161718192021222324252627282930

Fraction No. $\quad$ Fraction No.

\begin{tabular}{lll|l|l|l|l|l|l|l}
123456789101112131415 & 123456789101112131415
\end{tabular}

Figure 3. Cleavage of oligonucleotides containing 5-fluorodeoxyuridine-monophosphate and TFT-monophosphate residues by whole-cell extracts of HeLa cells obtained through gel filtration. Whole-cell extracts of HeLa cells were prepared as described in Materials and methods and the extracts were loaded on a Superdex 75 column $(1.0 \times 30 \mathrm{~cm})$ at a flow rate of $0.3 \mathrm{ml} / \mathrm{min}$. Fractions were collected from the void volume (No. 1) to the column volume (No. 15) at $2 \mathrm{~min} /$ tube. Each of the fractions was reacted with 5FU/A (lanes 1-15) or 5FU/G (lanes 16-30) containing oligonucleotides at $37^{\circ} \mathrm{C}$ for $2 \mathrm{~h}$ (A). Each of the fractions was also reacted with TFT/A (lanes 1-15) or TFT/G (lanes 16-30) containing oligonucleotides at $37^{\circ} \mathrm{C}$ for $2 \mathrm{~h}$ (B) or $10 \mathrm{~h}(\mathrm{C})$. 
D
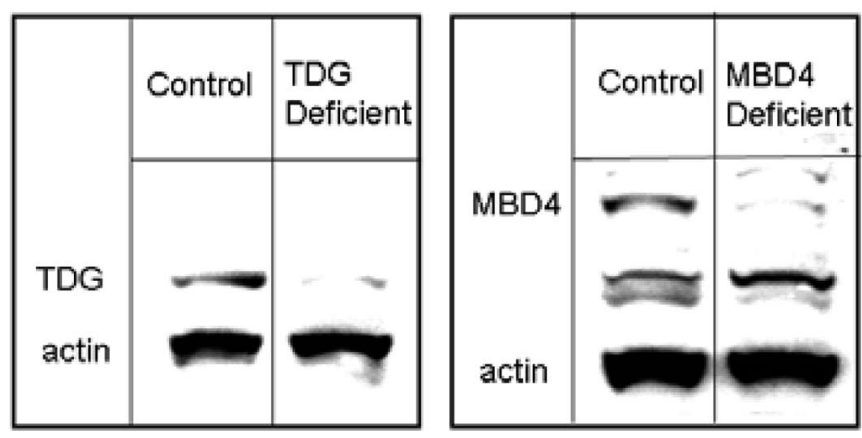

(Fig. 3). DNA glycosylase in the fractionated cell extracts was identified by the molecular weight, and immunoprecipitation (Fig. 3D-F). When a double-stranded oligomer containing $5 \mathrm{FU} / \mathrm{A}$ or $5 \mathrm{FU} / \mathrm{G}$ base pairs were reacted with the fractionated HeLa cell extracts for $2 \mathrm{~h}$ at room temperature, excision activities for the 5FU/A base pair were observed in the UDG-fraction (No. 7-10) and SMUG1-fraction (No. 8-11), and for the 5FU/G base pair were observed in MBD4-fraction (No. 1-4), TDGfraction (No. 6-8), UDG-fraction (No. 7-10), and SMUG1-fraction (No. 8-11) (Fig. 3A). These results seemed similar to the previously reported properties of the DNA glycosylases for double-stranded oligomers containing 5FU (8-10).

$\mathrm{E}$
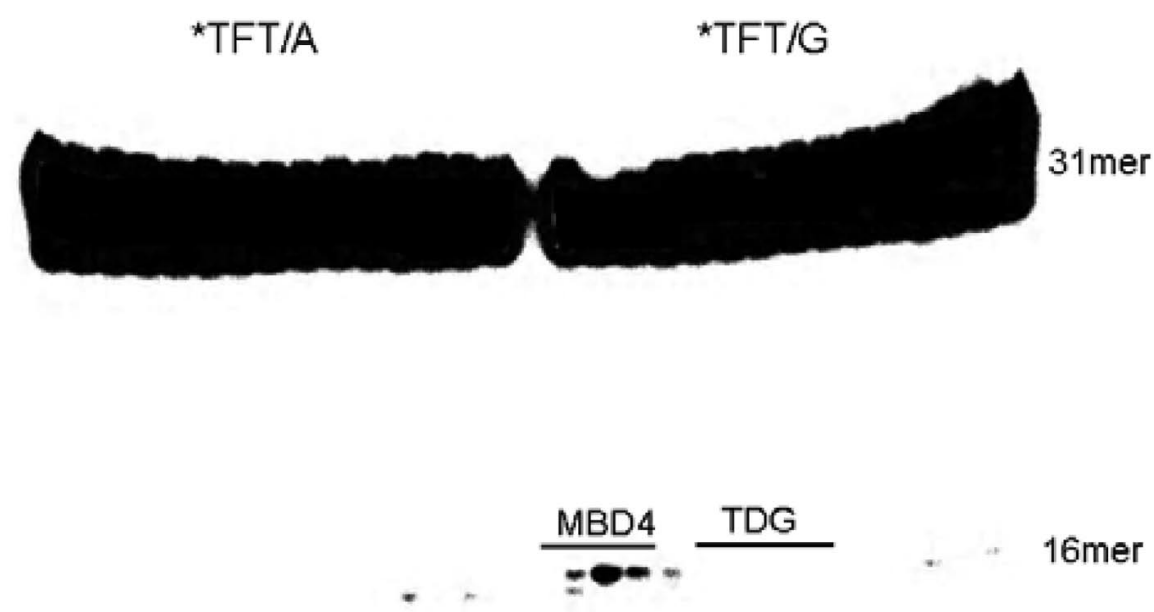

Lane No.

123456789101112131415161718192021222324252627282930

Fraction No. $\quad$ Fraction No.

123456789101112131415123456789101112131415

F

*TFT/A *TFT/G

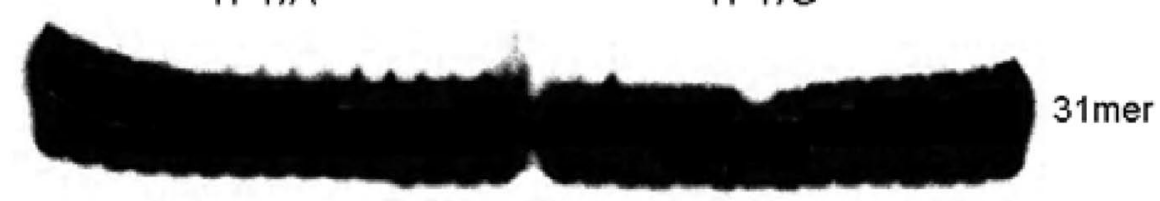

$\underline{\text { MBD4 }} \frac{\text { TDG }}{-9}$

$16 \mathrm{mer}$

Lane No.

123456789101112131415161718192021222324252627282930

Fraction No. $\quad$ Fraction No.

\begin{tabular}{llllll|lll}
123456789101112131415 & 123456789101112131415
\end{tabular}

Figure 3 (continued). Western blot analysis of whole-cell extracts of HeLa cells depleted of TDG or MBD4 by immunoprecipitation methods are indicated (D). Whole-cell extracts of HeLa cells depleted of TDG (E) or MBD4 (F) were loaded on gel filtration columns. Each fraction was reacted at $37^{\circ} \mathrm{C}$ for $10 \mathrm{~h}$. 
A

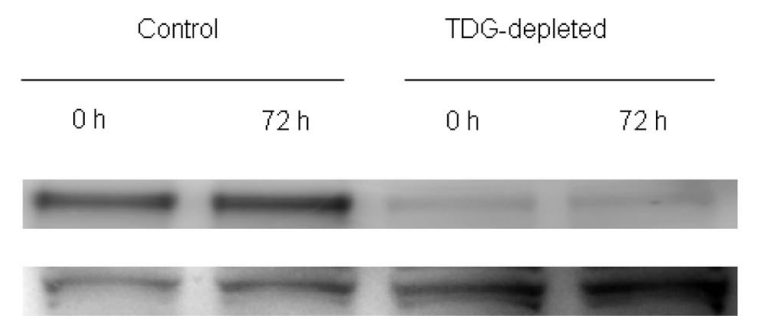

TDG

B

\begin{tabular}{|c|c|c|c|}
\hline \multicolumn{2}{|c|}{ Control } & \multicolumn{2}{|c|}{ MBD4-depleted } \\
\hline $\mathrm{Oh}$ & $72 \mathrm{~h}$ & on & $72 h$ \\
\hline
\end{tabular}

MBD4

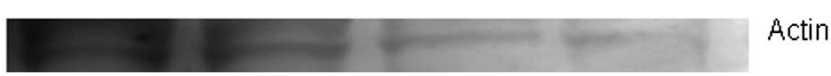

C

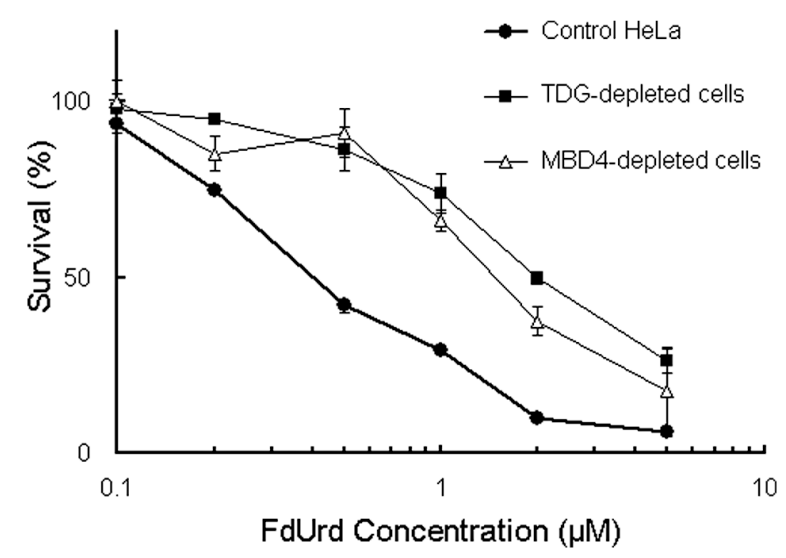

$\mathrm{D}$

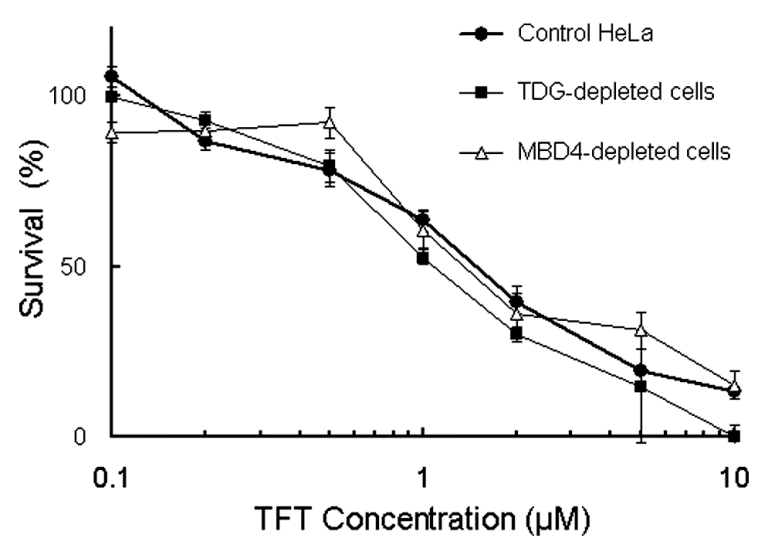

Figure 4. Cytotoxicity of TDG- or MBD4-depleted HeLa cells treated with FdUrd or TFT. Western blot analysis was performed for non-silencing shRNA HeLa cells (control), TDG-targeted knockdown HeLa cells (TDG-depleted) (A), and MBD4-targeted knockdown HeLa cells (MBD4-depleted) (B) at the 0 and $72 \mathrm{~h}$ time-points. The results of MTT assays of the control (•), TDGdepleted (๘), and MBD4-depleted $(\triangle)$ HeLa cells treated with FdUrd (C) or TFT (D) are indicated. Points are mean of $n=6$, and bars are SE.
Under the same condition, when a double-stranded oligomer containing a TFT/A or TFT/G base pair was reacted with each of the fractionated HeLa cell extracts, no excision activity was observed (Fig. 3B). However, when the incubation times were prolonged from 2 to $10 \mathrm{~h}$ at room temperature, excision activities were detected in the MBD4-fraction and TDG-fraction (Fig. 3C). These results indicate that MBD4 and TDG were able to recognize and excise TFT paired to guanine in double-stranded DNA, although this activity was weaker than that of $5 \mathrm{FU}$. This was further confirmed by an experiment using MBD4-depleted or TDG-depleted wholecell extracts of HeLa cells using an immunoprecipitation method. When a TDG-depleted HeLa cell extract was loaded through the gel filtration column, excision activities were observed in one major peak only; the fraction 5-8 peak disappeared (Fig. 3E). On the contrary, when the experiment was performed using an MBD4-depleted HeLa whole-cell extracts, the fraction 1-4 peak disappeared (Fig. 3F).

Cytotoxicity of TFT and FdUrd-treated HeLa cells in the presence of different protein levels of TDG and MBD4. Western blot analysis of TDG and MBD4. Since it was still unclear as to whether the enzymatic activities of TDG and MBD4 might contribute to the cytotoxicity of TFT and FdUrd, a MTT assay of cells treated with TFT or FdUrd was performed using small-interfering RNA-mediated knockdown of TDG or MBD4. Fig. 4A and B indicates that the results of Western blotting of the cells treated with non-silencing shRNA or shRNA targeting TDG or MBD4, respectively.

shRNA knockdown of HeLa cell TDG and MBD4 decreases the sensitivity of these cells to the effects of FdUrd. At first, we investigated whether knockdown of TDG or MBD4 might lead to a decrease in the sensitivity of the cells to FdUrd (Fig. 4C). The results of these experiments showed that knockdown of both TDG and MBD4 led to a significant increase in the resistance to the cytotoxic effects of FdUrd. The $\mathrm{IC}_{50}$ value increased by $>3$-fold for the cells with shRNA-mediated knockdown of TDG [2.11 $\mu \mathrm{M}(95 \% \mathrm{CI}, 1.72-2.50)]$ or MBD4 [1.57 $\mu \mathrm{M}(95 \% \mathrm{CI}, 1.00-2.14)]$ as compared with that for the cells treated with non-silencing shRNA $[0.45 \mu \mathrm{M}(95 \% \mathrm{CI}$, 0.21-1.05)]. These present data on the sensitivity to FdUrd are consistent with previous reports (15-17).

shRNA knockdown of HeLa TDG and MBD4 did not decrease the sensitivity of these cells to the effects of TFT. Under the same conditions, the sensitivity of the TDG- and MBD4-depleted HeLa cells to TFT was also determined (Fig. 4D). The results, shown in Fig. 4D, revealed no significant difference in the sensitivity to TFT between the wild-type HeLa cells and the TDG- or MBD4-depleted cells. The $\mathrm{IC}_{50}$ value was $1.15 \mu \mathrm{M}$ (95\% CI, 0.95-1.36) for the TDG-depleted HeLa cells, $1.40 \mu \mathrm{M}$ (95\% CI, 0.55-3.35) for the MBD4-depleted HeLa cells, and $1.47 \mu \mathrm{M}(95 \% \mathrm{CI}, 1.25-1.71)$ for the wild-type HeLa cells.

\section{Discussion}

Trifluorothymidine (TFT) was first synthesized by Heidelberger et al, in 1964 (18), they had already shown that TFT, similar to the case of 5-fluoro-2'-deoxyuridine (FdUrd), is incorporated 
into the cellular DNA to prevent cell division, in contrast to thymidine, 5-bromodeoxyuridine and 5-iododeoxyuridine, which support cell division (19). Comparison of the $\mathrm{IC}_{50}$ values of several nucleosides against HeLa cells revealed that the uptake of TFT into the cellular DNA [62.22 for $4 \mathrm{~h}$ (pmol/1x $10^{6}$ cells)] was significantly greater than that of $5 \mathrm{FU}$ [0.17 for $4 \mathrm{~h}$ ( $\mathrm{pmol} / 1 \times 10^{6}$ cells)] or FdUrd [7.53 for $4 \mathrm{~h}(\mathrm{pmol} / 1$ $\mathrm{x} 10^{6}$ cells)], as described in Table I. These results suggest that TFT may have more direct effects on the cellular DNA than 5 FU or FdUrd.

We investigated the effect of TFT triphosphate (TFT-TP) against DNA polymerase $\alpha$ (Fig. 2). In an experiment conducted by the addition of increasing concentrations of TFT-TP in $4 \mathrm{~N}$ reactions, no competitive inhibition by TFT-TP of elongation induced by dTTP was observed, even at the maximal concentration of $100 \mu \mathrm{M}$. Therefore, DNA polymerases are probably not the major targets, because, as indicated above, TFT-TP competed weakly with dTTP in producing direct inhibition of DNA polymerases. Since TFT inhibits TS, reduction of the cellular dTTP may potentiate the incorporation of TFT into the cellular DNA. Massive incorporation of TFT into the DNA may, however, account for the potent inhibitory activity of TFT on the DNA synthesis in whole cells.

It has been argued that the therapeutic effects of TS inhibition are based on the fragmentation of genomic DNA as a result of massive uracil excision by the replication-associated uracil DNA glycosylase, which connected with futile cycles of base excision repair (BER) (20). Although TFT also has an inhibitory effect against TS, it is nearly impossible to explain by its BER futile cycling model, which dUTP/dTTP imbalance gives rise to the misincorporation of dUMP during DNA replication, because TFT-TP itself is incorporated into the DNA to a much greater extent than 5FU and FdUrd (Table I).

Cells deficient in the TDG (15) and MBD4 (a.k.a. MED1) $(16,17)$ DNA glycosylases have been reported to be resistant to $5 \mathrm{FU}$. These observation would appear to contradict the hypothesis that $5 \mathrm{FU}$ incorporated in the DNA is toxic. However, Kunz et al (15) reported that excision of DNA-incorporated 5FU by TDG generates persistent DNA strand breaks, delays S-phase progression, and activates DNA damage signaling. Therefore, a deficiency of TDG may also result in an impaired cell death response, leading to the observed resistance of the cells to 5FU exposure. On the other hand, a deficiency of MBD4 may also result in an impaired mismatch repair-dependent cell death response, leading to the observed resistance of the cells to 5FU exposure $(16,17)$. These present data on the sensitivity to FdUrd are consistent with those reported previously (Fig. 4C). On the contrary, there was no significant difference in the sensitivity to TFT between wild-type HeLa cells and TDG- or MBD4-depleted HeLa cells (Fig. 4D).

First, almost all of the TFT is incorporated into the T-sites (T/A, not T/G base pair) of the cellular DNA. The primer extension assays demonstrated that TFT-TP has a $\mathrm{Km}$ value very similar to that of dTTP (data not shown), and that adenine is incorporated precisely into the TFT lesion in the template (Fig. 1B). In this case, TFT incorporated into the DNA may be removed by other repair pathways than BER, because TFT induces DNA fragmentation (7), and arrest of the G2/M-phase of the cell cycle $(21,22)$.
Second, although misincorporated TFT paired to guanine in the DNA may be removed by TDG and MBD4, its repair is not mediated with mechanism of cancer cell death by TFT unlike 5FU and FdUrd (15-17). The substitution of a thymidine residue for a TFT residue causes a slight decrease in the DNA duplex stability (23), although DNA double helices are still formed that adopt the B-form conformation (24). Thus, cell death on cancer cells may be executed by differential mechanisms between TFT and 5FU.

In conclusion, TFT is incorporated to a significantly greater degree into the DNA than either 5FU or FdUrd, however, it completed only weakly with thymidine triphosphate. The DNA glycosylase assay indicated that there was no detectable excision of TFT paired to adenine by any of the uracil DNA glycosylases (UDG), thymine DNA glycosylase (TDG), methyl-CpG binding domain 4 (MBD4), and fractions of HeLa cell extracts, while TDG and MBD4 were able to excise TFT paired to guanine. However, small-interfering RNA-mediated knockdown of TDG or MBD4 did not significantly increase the cellular resistance to the cytotoxic effects of TFT. Therefore, these data suggest that the inhibitory effects of TFT on DNA replication and repair enzymes are apparently distinct from those of 5FU and FdUrd. Such antimetabolites with novel mechanisms of action could complement current chemotherapeutic agents for the treatment of human cancers, especially by suppressing the appearance of drug resistance.

\section{References}

1. Glazer RI and Lloyd LS: Association of cell lethality with incorporation of 5-fluorouracil and 5-fluorouridine into nuclear RNA in human colon carcinoma cells in culture. Mol Pharmacol 21: 468-473, 1982.

2. Peters GJ, Backus HH, Freemantle S, van Triest B, CodacciPisanelli G, van der Wilt CL, Smid K, Lunec J, Calvert AH, Marsh S, McLeod HL, Bloemena E, Meijer S, Jansen G, van Groeningen CJ and Pinedo HM: Induction of thymidylate synthase as a 5-fluorouracil resistance mechanism. Biochim Biophys Acta 1587: 194-205, 2002.

3. Robins QAnP, Lindahl T and Barnes DE: 5-Fluorouracil incorporated into DNA is excised by the Smug1 DNA glycosylase to reduce drug cytotoxicity. Cancer Res 67: 940-945, 2007.

4. Umeda $M$ and Heidelberger C: Comparative studies of fluorinated pyrimidines with various cell lines. Cancer Res 28: 2529-2538, 1968.

5. Murakami Y, Kazuno H, Emura T, Tsujimoto H, Suzuki N and Fukushima M: Different mechanisms of acquired resistance to fluorinated pyrimidines in human colorectal cancer cells. Int J Oncol 17: 277-283, 2000.

6. Emura T, Nakagawa F, Fujioka A, Ohshimo H, Yokogawa T, Okabe $\mathrm{H}$ and Kitazato $\mathrm{K}$ : An optimal dosing schedule for a novel combination antimetabolite, TAS-102, based on its intracellular metabolism and its incorporation into DNA. Int J Mol Med 13: 249-255, 2004.

7. Emura T, Suzuki N, Yamaguchi M, Ohshimo H and Fukushima M: A novel combination antimetabolite, TAS-102, exhibits antitumor activity in FU-resistant human cancer cells through a mechanism involving FTD incorporation in DNA. Int J Oncol 25: 571-578. 2004.

8. Kavli B, Sundheim O, Akbari M, Otterlei M, Nilsen H, Skorpen F, Aas PA, Hagen L, Krokan HE and Slupphaug G: hUNG2 is the major repair enzyme for removal of uracil from U:A matches, U:G mismatches, and U in single-stranded DNA, with hSMUG1 as a broad specificity backup. J Biol Chem 277: 39926-39936, 2002.

9. Cortazar D, Kunz C, Saito Y, Steinacher R and Schar P: The enigmatic thymine DNA glycosylase. DNA Repair (Amst) 6: 489-504, 2007. 
10. Wong E, Yang K, Kuraguchi M, Werling U, Avdievich E, Fan K, Fazzari M, Jin B, Brown AM, Lipkin M and Edelmann W: Mbd4 inactivation increases Cright-arrowT transition mutations and promotes gastrointestinal tumor formation. Proc Natl Acad Sci USA 99: 14937-14942, 2002.

11. Suzuki N and Fukushima M: Simple and rapid enzymatic method for the synthesis of single-strand oligonucleotides containing trifluorothymidine. Nucleosides Nucleotides Nucleic Acids 29: 896-904, 2010.

12. Manley JL, Fire A, Cano A, Sharp PA and Gefter ML: DNAdependent transcription of adenovirus genes in a soluble whole-cell extract. Proc Natl Acad Sci USA 77: 3855-3859, 1980.

13. Mikita T and Beardsley GP: Functional consequences of the arabinosylcytosine structural lesion in DNA. Biochemistry 27 4698-4705, 1988.

14. Neddermann $P$ and Jiricny $\mathrm{J}$ : The purification of a mismatchspecific thymine-DNA glycosylase from HeLa cells. J Biol Chem 268: 21218-21224, 1993 .

15. Kunz C, Focke F, Saito Y, Schuermann D, Lettieri T, Selfridge J and Schar P: Base excision by thymine DNA glycosylase mediates DNA-directed cytotoxicity of 5-fluorouracil. PLoS Biol 7: 967-979, 2009.

16. Cortellino S, Turner D, Masciullo V, Schepis F, Albino D, Daniel R, Skalka AM, Meropol NJ, Alberti C, Larue L and Bellacosa A: The base excision repair enzyme MED1 mediates DNA damage response to antitumor drugs and is associated with mismatch repair system integrity. Proc Natl Acad Sci USA 100: 15071-15076, 2003

17. Sansom OJ, Zabkiewicz J, Bishop SM, Guy J, Bird A and Clarke AR: MBD4 deficiency reduces the apoptotic response to DNA-damaging agents in the murine small intestine. Oncogene 22: 7130-7136, 2003.
18. Heidelberger C, Parsons DG and Remy DC: Syntheses of 5-trifluoromethyluracil and 5-trifluoromethyl-2'-deoxyuridine. J Med Chem 7: 1-5, 1964

19. Fujiwara Y, Oki T and Heidelberger C: Fluorinated pyrimidines. XXXVII. Effects of 5-trifluoromethyl-2'-deoxyuridine on the synthesis of deoxyribonucleic acid of mammalian cells in culture. Mol Pharmacol 6: 273-280, 1970.

20. Longley DB, Harkin DP and Johnston PG: 5-fluorouracil: mechanisms of action and clinical strategies. Nat Rev Cancer 3: 330-338, 2003.

21. Temmink OH, Hoebe EK, Fukushima M and Peters GJ: Irinotecaninduced cytotoxicity to colon cancer cells in vitro is stimulated by pre-incubation with trifluorothymidine. Eur J Cancer 43: 175-183, 2007.

22. Bijnsdorp IV, Kruyt FA, Gokoel S, Fukushima M and Peters GJ: Synergistic interaction between trifluorothymidine and docetaxel is sequence dependent. Cancer Sci 99: 2302-2308, 2008.

23. Markley JC, Chirakul P, Sologub D and Sigurdsson ST: Incorporation of 2'-deoxy-5-(trifluoromethyl)uridine and 5-cyano-2'-deoxyuridine into DNA. Bioorg Med Chem Lett 11: 2453-2455, 2001.

24. Gmeiner WH, Rao KE, Rayner B, Vasseur JJ, Morvan F, Imbach JL and Lown JW: Polarity of annealing and structural analysis of the RNase $\mathrm{H}$ resistant alpha-5'-d[TACACA].beta-5'r[AUGUGU] hybrid determined by high-field $1 \mathrm{H}, 13 \mathrm{C}$, and 31P NMR analysis. Biochemistry 29: 10329-10341, 1990. 